\title{
Influence of grating parameters on the linewidths of external-cavity diode lasers
}

\author{
Huanqian Loh, Yu-Ju Lin, Igor Teper, Marko Cetina, Jonathan Simon, James K. Thompson, \\ and Vladan Vuletić
}

\begin{abstract}
We investigate experimentally the influence of the grating reflectivity, grating resolution, and diode facet antireflection (AR) coating on the intrinsic linewidth of an external-cavity diode laser built with a diffraction grating in a Littrow configuration. Grating lasers at 399, 780, and $852 \mathrm{~nm}$ are determined to have typical linewidths between 250 and $600 \mathrm{kHz}$ from measurements of their frequency noise power spectral densities. The linewidths are little affected by the presence of an AR coating on the diode facet but narrow as the grating reflectivity and grating resolution are increased, with the resolution exerting a greater effect. We also use frequency noise measurements to characterize a laser mount with improved mechanical stability. (C) 2006 Optical Society of America

OCIS codes: $\quad 140.2020,140.3600,050.1950$.
\end{abstract}

\section{Introduction}

Diode lasers have vast applications in atomic physics, because of their reliability, easy tunability, and low cost. ${ }^{1,2}$ Diode lasers alone, however, have linewidths of $\sim 20 \mathrm{MHz},{ }^{3}$ which are often too broad for manipulating atoms and are typically not tunable to every wavelength of interest. Optical feedback, achieved by adding an external-cavity element such as a diffraction grating, not only enhances the wavelength tunability, but also reduces the intrinsic linewidth to below $1 \mathrm{MHz}{ }^{1-3}$

Linewidths of external-cavity diode lasers have been extensively studied both theoretically ${ }^{4-13}$ and experimentally. ${ }^{14-19}$ Theoretical analysis shows that to lowest order, the linewidth of an external-cavity laser is reduced from its original diode chip linewidth $\Delta \nu_{0}$ by the factor $\left[\tau_{d} /\left(\tau_{d}+\tau_{e}\right)\right]^{2}$, where $\tau_{d}$ and $\tau_{e}$ are the round-trip times for the diode and the external cavity, respectively. ${ }^{9}$ A long external cavity can therefore yield a very narrow intrinsic linewidth at the expense

H. Loh (huanqian@alum.mit.edu), Y.-J. Lin, I. Teper, M. Cetina, J. K. Thompson, and V. Vuletić are with the Department of Physics, MIT-Harvard Center for Ultracold Atoms, and Research Laboratory of Electronics, Massachusetts Institute of Technology, Cambridge, Massachusetts 02139. J. Simon is with the Department of Physics, MIT-Harvard Center for Ultracold Atoms, Harvard University, Cambridge, Massachusetts 02138.

Received 27 March 2006; revised 11 July 2006; accepted 18 August 2006; posted 22 August 2006 (Doc. ID 69329).

$0003-6935 / 06 / 369191-07 \$ 15.00 / 0$

(C) 2006 Optical Society of America of the mechanical stability and frequency tuning range. Since strong atomic transitions have typical linewidths of several megahertz, it is usually sufficient to work with external cavities that are a few centimeters long, which generate linewidths of several hundred kilohertz.

With a fixed external-cavity length, one can further reduce the intrinsic linewidth by fine-tuning the mechanical and optical designs of the external cavity. Most theoretical models use a simplified approach in that they analyze a cavity formed by an external mirror rather than a grating, and consider only a single longitudinal mode, predicting that the linewidth narrows as the mirror reflectivity is increased. On the other hand, competition between multiple diode chip longitudinal modes has been suggested as a mechanism for linewidth broadening. ${ }^{4,20}$ In that case, the linewidth should also depend on how well neighboring diode chip modes can be suppressed by the grating spectral profile, as characterized by the grating resolution $\lambda / \Delta \lambda=m N$, where $m$ is the diffraction order, and $N$ is the number of illuminated grating lines.

To the best of our knowledge, the effect of the grating resolution on the laser linewidth has not previously been investigated experimentally. Furthermore, while an antireflection (AR) coating on the front facet of the laser diode is known to improve wavelength tunability, its effect on the linewidth is less clear. Wyatt has reported a significant linewidth dependence on the AR coating of a $1.5 \mu \mathrm{m}$ laser, ${ }^{16}$ while another experiment showed no effect 
for a $1.3 \mu \mathrm{m}$ InGaAsP laser. ${ }^{17}$ In the present work, we investigate how the linewidths of near-IR gratingtuned diode lasers (780 $\mathrm{nm}$ for $\mathrm{Rb}$ and $852 \mathrm{~nm}$ for Cs) and near-UV lasers (399 $\mathrm{nm}$ for $\mathrm{Yb}$ ) are influenced by the grating reflectivity, grating resolution, and diode AR coating. We find that the chip AR coating has little effect, while increasing the grating reflectivity at the expense of laser output power can modestly decrease the linewidth, in qualitative agreement with theoretical models. However, we find that a $50 \%$ increase in grating groove density reduces the linewidth by almost a factor of 2 . This indicates that competition between different longitudinal modes substantially contributes to the line broadening of external-cavity semiconductor lasers and needs to be included into realistic models.

If a reference laser of sufficiently narrow linewidth and high-frequency stability is available, the test laser linewidth can be measured directly by a heterodyne measurement on a fast photodiode, which yields the convolution of the spectral profiles of the two lasers. In this study, we have instead obtained the linewidth by measuring the power spectral density $S(f)$ of the test laser's frequency noise fluctuations. $S(f)$ is expected to display higher noise at low Fourier frequencies due to mechanical vibrations. The noise then falls until it reaches a white-noise level $S_{0}$, at high Fourier frequencies. ${ }^{21} S_{0}$ is related to the intrinsic Lorentzian linewidth $\Delta v$ by $^{22}$

$$
\Delta v=\pi S_{0}^{2} .
$$

To convert frequency noise into intensity noise that can be measured with a photodiode, either the transmission signal from a Fabry-Perot cavity ${ }^{23,24}$ or the atomic resonance line ${ }^{21}$ can be used, where the latter offers the advantage of being insensitive to mechanical vibrations. Using the atomic line to measure noise at low Fourier frequencies, we have also characterized a laser mount with improved mechanical properties, a schematic of which is presented in Section 4.

\section{Frequency Noise Measurements}

A typical grating laser used in this study is assembled in a Littrow configuration. ${ }^{2}$ We carefully optimize its collimation and grating alignment to achieve the best possible optical feedback using the following procedure: monitoring the laser optical power, we set the laser diode current to just below its threshold value. When the current is dithered, the onset of lasing manifests as a sudden increase of optical power with the current. We assume that the feedback is optimized when the threshold is minimized by adjusting the collimator position and the grating angle. The feedback is much more sensitive to the collimator position than to the grating angle. ${ }^{21,25}$

Figure 1 shows how the wavelength of an ARcoated laser tunes with the current for both optimized and slightly misaligned feedback. When the feedback is optimized, the wavelength tunes smoothly with

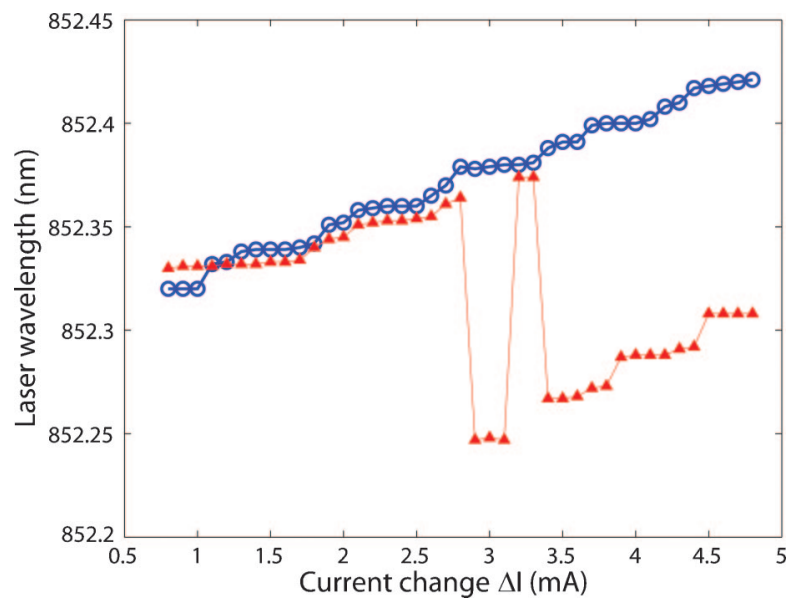

Fig. 1. (Color online) Tuning behavior of laser wavelength with current, for optimized (open circles) and nonoptimized (filled triangles) feedback, measured for an $852 \mathrm{~nm}$ laser diode with an AR coating.

laser current, with small jumps of $4.5 \mathrm{GHz}$ between external-cavity modes. On the other hand, when the vertical grating angle or the collimator lens is slightly misaligned, large frequency jumps of $48 \mathrm{GHz}$ appear, corresponding to lasing on different residual diode chip modes that have not been completely suppressed by the AR coating.

Once the feedback is optimized, the laser light is sent through an atomic cell and onto an avalanche photodiode with a bandwidth of $300 \mathrm{kHz}$. This bandwidth is sufficient to measure the intrinsic laser linewidth, because the white frequency noise typically appears from Fourier frequencies of $20 \mathrm{kHz}$ onward. Figure 2 shows a schematic of the setup used to measure the laser frequency noise. The laser wavelength is tuned to a value that matches the slope of a Doppler absorption profile (e.g., $852.33 \mathrm{~nm}$ for Cs), so that laser frequency noise is converted into amplitude noise for detection by the avalanche photodiode. For the $399 \mathrm{~nm}$ laser, the dichroic atomic vapor laser lock dispersive signal from a hollow cathode lamp (Hamamatsu L2783-70HE-Yb) is used instead. ${ }^{26}$

The slope of the absorption line is calibrated by heterodyning the grating laser with a reference laser locked to another atomic cell. The slopes on the two sides of the absorption line are observed to be asym-

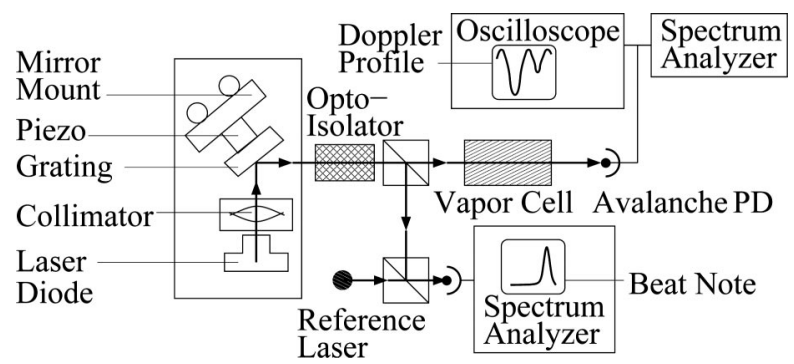

Fig. 2. Schematic of the setup used to measure laser frequency noise. Components such as attenuators are not drawn. The polarization of the laser light is out of the plane. 
metric because of the linear increase in laser output power with the wavelength. To remove this linear dependence, we average over measurements on both slopes of the atomic line.

The noise as measured by the avalanche photodiode in Fig. 2 contains both frequency and amplitude noises. We measure the amplitude noise by removing the atomic cell and inserting gray filters to attenuate the beam power to its previous value on the photodiode. We find that the amplitude noise scales approximately as the square root of the power incident on the photodiode, an indication that the amplitude noise at the frequencies of interest is dominated by photon shot noise, in agreement with a calculation using the known photodiode gain. Figure 3 shows both the total noise and the amplitude noise contributions for a typical measurement. The frequency noise is obtained by subtracting the uncorrelated amplitude noise from the total noise in quadrature. The noise below $5 \mathrm{kHz}$ generally reflects mechanical vibrations of the laser mount, whereas the noise above $5 \mathrm{kHz}$ becomes approximately independent of frequency, indicating a Lorentzian line shape. ${ }^{22}$

Applying Eq. (1) to the white-noise portion, we measure typical linewidths of the 780 and $852 \mathrm{~nm}$ grating lasers to be in the $250-600 \mathrm{kHz}$ range (see Tables 1 and 2), which is expected ${ }^{9}$ for diodes of length $L_{d}$ $\simeq 1 \mathrm{~mm}$ and an index of refraction of $n_{d}=3.5$, set in an external cavity of length $L_{e}=3.3 \mathrm{~cm}$. We also determine a similar linewidth of $250 \mathrm{kHz}$ for a $399 \mathrm{~nm}$ Nichia NDHV310APC laser diode (nonAR coated) assembled with a $2400 \mathrm{~mm}^{-1}$ grating of reflectivity $R_{G 1}=0.60$.

For comparison, the method of converting frequency into amplitude noise via the atomic absorption line has allowed us to measure a linewidth as narrow as $30 \mathrm{kHz}$ for a distributed Bragg reflector

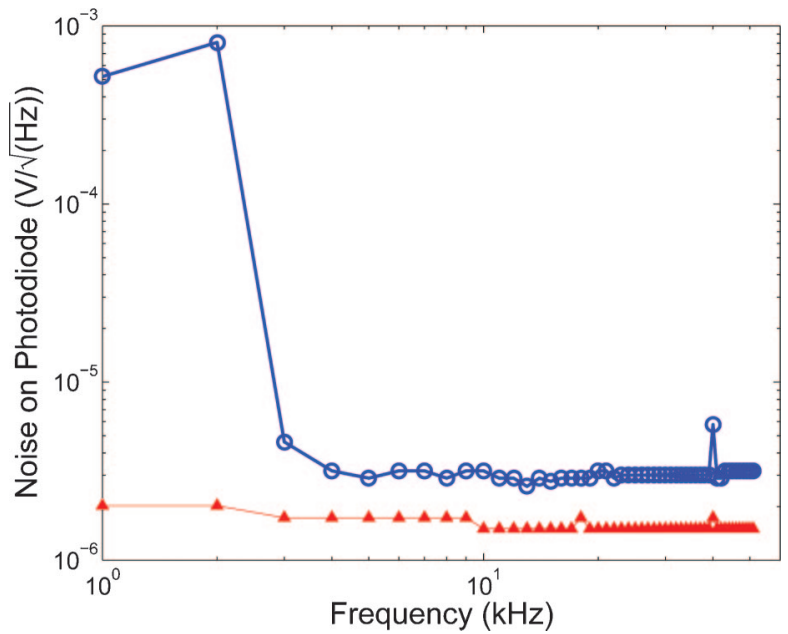

Fig. 3. (Color online) Total (open circles) and amplitude (filled triangles) spectral noise densities of an $852 \mathrm{~nm}$ AR-coated laser assembled with a $1200 \mathrm{~mm}^{-1}$ grating of reflectivity $R_{G 1}=0.21$. For the frequency noise, $1 \mathrm{~V} / \sqrt{\mathrm{Hz}}$ corresponds to $156 \mathrm{MHz} / \sqrt{\mathrm{Hz}}$. For the amplitude noise, $1 \mathrm{~V} / \sqrt{\mathrm{Hz}}$ corresponds to a fractional noise of $0.94 / \sqrt{\mathrm{Hz}}$.
Table 1. Linewidths of $852 \mathrm{~nm}$ Lasers for Different Gratings in the Littrow Configuration ${ }^{a}$

\begin{tabular}{cccccc}
\hline $\begin{array}{c}n \\
\left(\mathrm{~mm}^{-1}\right)\end{array}$ & $\lambda / \Delta \lambda$ & $R_{G 1}$ & $R_{G 0}$ & $\begin{array}{c}\Delta v_{\text {th }} \\
(\mathrm{kHz})\end{array}$ & $\begin{array}{c}\Delta \nu_{\exp } \\
(\mathrm{kHz})\end{array}$ \\
\hline 1200 & 4200 & 0.21 & 0.67 & $260,{ }^{b} 290,{ }^{c} 320^{d}$ & $560 \pm 140$ \\
1200 & 4200 & 0.61 & 0.19 & $260,{ }^{b} 270,{ }^{c} 290^{d}$ & $440 \pm 110$ \\
1800 & 8400 & 0.16 & 0.78 & $260,{ }^{b} 290,{ }^{c} 330^{d}$ & $320 \pm 60$ \\
\hline
\end{tabular}

${ }^{a}$ Linewidths of AR-coated $852 \mathrm{~nm}$ lasers built with gratings 43773, 43753, 43222 from Edmund Optics for lines 1-3. For a Littrow grating laser, the first diffraction order with power reflectivity $R_{G 1}$ is reflected back into the laser for optical feedback, while the zeroth order with power reflectivity $R_{G 0}$ is used as the laser output. The grating resolution $\lambda / \Delta \lambda$ is computed for a beam diameter of $D \simeq 3 \mathrm{~mm}$ from the groove density $n$ and the Littrow angle. The free-running laser linewidth is estimated to be $35 \mathrm{MHz}$ (Ref. 28). $\Delta v_{\text {th }}$ lists different theoretical predictions for the given laser parameters, and $\Delta v_{\exp }$ is the linewidth measured using Eq. (1).

${ }^{b} \Delta v_{\text {th }}=\Delta v_{0}\left[\tau_{d} /\left(\tau_{d}+\tau_{e}\right)\right]^{2}$.

${ }^{c} \Delta v_{\text {th }}=\Delta v_{0} /(1+A+B)^{2}$, where $A$ and $B$ are calculated from Eq. (26) of Kazarinov and Henry (Ref. 12).

${ }^{d}$ Calculated from Eq. (8) of the paper of Sun et al. (Ref. 10).

laser diode, whose intrinsic linewidth was narrowed by optical feedback from a low finesse optical cavity using a setup similar to that described by Dahmani et $a l .{ }^{27}$

\section{Effects of Grating Reflectivity, Grating Resolution, and Antireflection Coating on the Laser Linewidth}

Table 1 shows the linewidths measured for various gratings (Edmund Optics 43222, 43753, and 43773) assembled with the same AR-coated laser diode chip (Sacher Lasertechnik SAL-850-50, back facet reflectivity $R_{1}=0.85$, front facet reflectivity $R_{2}=10^{-4}$, length $L_{d}=890 \mu \mathrm{m}$, estimated intrinsic linewidth ${ }^{28}$ $\Delta \nu_{0}=35 \mathrm{MHz}$ ) and collimator (Thorlabs C390TM-B,

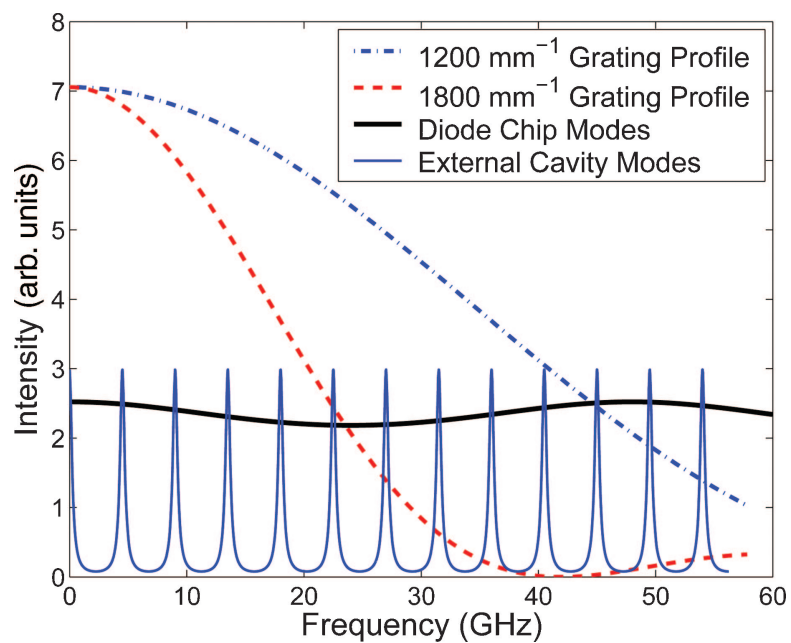

Fig. 4. (Color online) Calculated profiles of 1200 and $1800 \mathrm{~mm}^{-1}$ gratings, overlapped with diode-chip modes and external-cavity modes spaced by frequency intervals determined from Fig. 1 . The passive external-cavity mode spectrum is calculated for a grating of reflectivity $R_{G 1}=0.61$ and diode back facet reflectivity $R_{1}=0.85$. The diode chip mode spectrum is calculated for an AR-coated $\left(R_{2}=10^{-4}\right)$ diode with a regenerative gain parameterized by the material absorption coefficient $\alpha_{L}=45 \mathrm{~cm}^{-1}$ (Ref. 29). 
Table 2. Linewidths of Both Non-AR- and AR-Coated $780 \mathrm{~nm}$ Lasers ${ }^{a}$

\begin{tabular}{lccccc}
\hline \multicolumn{1}{c}{ Diode Model } & $\begin{array}{c}L_{d} \\
(\mu \mathrm{M})\end{array}$ & $R_{1}$ & $R_{2}$ & $\begin{array}{c}\Delta v_{\text {th }} \\
(\mathrm{kHz})\end{array}$ & $\begin{array}{c}\Delta v_{\text {exp }} \\
(\mathrm{kHz})\end{array}$ \\
\hline Sanyo DL7140-201S & 840 & 0.85 & 0.15 & $4900,{ }^{b} 130,^{c}-^{e}$ & $500 \pm 80$ \\
SAL-780-40 & 890 & 0.85 & $8 \times 10^{-5}$ & $43,000,{ }^{b} 260,{ }^{c} 370^{d}$ & $450 \pm 80$ \\
\hline
\end{tabular}

${ }^{a}$ Linewidths of $780 \mathrm{~nm}$ lasers without and with an AR coating on the diode front facet, assembled with the same grating (Edmund Optics 43773). $L_{d}$ is the diode chip length, while $R_{1}$ and $R_{2}$ are the back and front facet power reflectivities, respectively. $\Delta v_{\text {th }}$ and $\Delta v_{\text {exp }}$ are as defined in Table 1.

${ }^{b}$ Reference 28.

${ }^{c} \Delta v_{\text {th }}=\Delta v_{0} /(1+A+B)^{2}$, where $A$ and $B$ are calculated from Eq. (26) of Kazarinov and Henry's paper (Ref. 12).

${ }^{d}$ Calculated from Eq. (8) of the paper of Sun et al. (Ref. 10).

${ }^{e}$ The calculations of Sun et al. do not apply for $R_{2} / R_{G 1} \sim 1$ (Ref. 10).

effective focal length $=2.75 \mathrm{~mm}$ ). Different theoretical predictions for the reduced linewidth in the strong feedback regime are also given in Table 1.10,12

As expected from theory, ${ }^{10,12}$ the measured linewidth narrows somewhat with higher grating reflectivity $R_{G 1}$ for a fixed grating resolution, i.e., a linewidth decrease by $20 \%$ is observed as the reflectivity is tripled from $R_{G 1}=0.2$ to $R_{G 1}=0.6$. Surprisingly, a much larger effect is observed when the grating resolution is changed: the linewidth decreases by $40 \%$ as the resolution is doubled even when the grating reflectivity is lowered. The effect of the grating resolution shows that the simple mirror theory $\mathrm{y}^{4-10}$ is not fully adequate, and strongly suggests that for describing the linewidth of externalcavity lasers, the competition between diode chip modes must be taken into account. ${ }^{18}$ Table 1 also shows that the preferred method of decreasing the linewidth is to increase the grating resolution rather than the grating's first-order reflectivity, since the former does not decrease the available laser output power.

Figure 4 gives a physical picture accounting for both effects of grating reflectivity and resolution. The external cavity, formed between the back facet of the laser diode chip and the reflective grating surface, has a finesse determined by the first-order reflectivity of the grating. The grating resolution, on the other hand, sets the width of the grating profile in Fig. 4. Higher grating resolution, achieved with a grating of higher groove density or a collimator that produces larger beam size, suppresses neighboring diode chip modes better, leading to less mode competition and hence to less frequency noise. In fact, the greater influence of the grating resolution on the linewidth indicates that the suppression of other laser diode chip modes is more important than the finesse of the external cavity.

The results in Table 1 imply that a narrower linewidth can be obtained without sacrificing laser output power by simply using a grating of higher groove density. In addition, we have attempted to increase the grating resolution by using a collimator that produced a larger beam size (Thorlabs C240TM-B, effective focal length $=8.0 \mathrm{~mm}$ ). We found that even when the optical feedback was only near optimized, we could already achieve a linewidth of $425 \mathrm{kHz}$ using the $1800 \mathrm{~mm}^{-1}$ grating. On the other hand, the com- bination of the larger beam size and the $1800 \mathrm{~mm}^{-1}$ grating also means that the optical feedback is much more sensitive to the grating angle. At such high sensitivity, slight thermal drifts of the aluminum laser mount made it extremely difficult for the grating to remain at its optimal angle. As a result, we were unable to maintain reliable operation in the mechanical setup of Fig. 2 for this collimator-grating combination.

We also study the effect of the diode AR coating on the linewidth. Table 2 shows both measured and calculated linewidths of AR-coated $\left(R_{2}=8 \times 10^{-5}\right)$ and AR-uncoated $\left(R_{2}=0.15\right)$ laser diodes assembled with the same grating $\left(R_{G 1}=0.27, n=1200 \mathrm{~mm}^{-1}\right)$. Henry's model predicts that the broader diode chip linewidth of an AR-coated laser ${ }^{28}$ compensates for its larger linewidth reduction factor ${ }^{12}$ when set in an external cavity, yielding a linewidth on the same order as a non-AR-coated diode laser used with the same grating. The $130 \mathrm{kHz}$ difference between the calculated linewidths in Table 2 is not necessarily significant, because the theory employed does not account for other diode chip modes, which would have been better suppressed in the AR-coated laser.

Table 2 shows a slightly narrower linewidth for the AR-coated laser, although the difference is less than the estimated error of $80 \mathrm{kHz}$ associated with each measurement. We conclude that although the AR coating eases the procedure for optimizing grating alignment as well as enhances wavelength tunability (Fig. 1), its effect on the linewidth is insignificant.

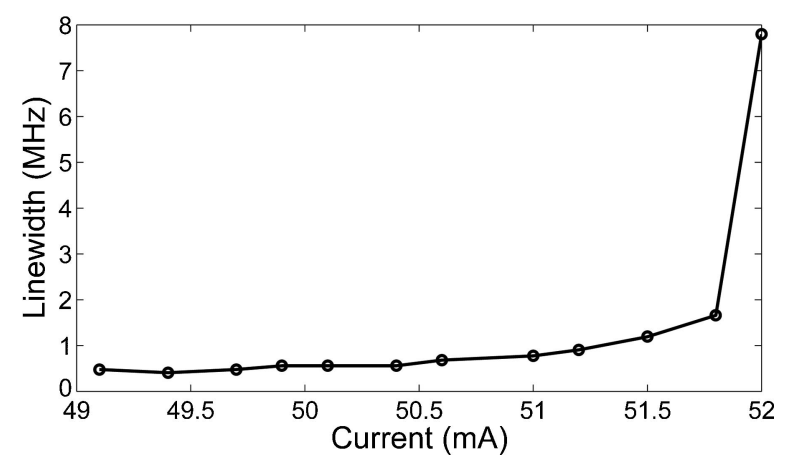

Fig. 5. Plot of linewidth versus current injected into the diode laser. The laser jumps to a different longitudinal cavity mode near $52 \mathrm{~mA}$. 
Table 3. Best Achieved Linewidths ${ }^{a}$

\begin{tabular}{|c|c|c|c|c|c|c|}
\hline Atoms & $\begin{array}{c}\lambda \\
(\mathrm{nm})\end{array}$ & $\begin{array}{c}\mathrm{AR} \\
\text { Coating }\end{array}$ & $\begin{array}{c}n \\
\left(\mathrm{~mm}^{-1}\right)\end{array}$ & $\lambda / \Delta \lambda$ & $R_{G 1}$ & $\begin{array}{l}\Delta v_{\exp } \\
(\mathrm{kHz})\end{array}$ \\
\hline $\mathrm{Cs}$ & 852 & Yes & 1800 & 8400 & 0.16 & $320 \pm 60$ \\
\hline $\mathrm{Rb}$ & 780 & Yes & 1200 & 4100 & 0.27 & $450 \pm 80$ \\
\hline $\mathrm{Yb}$ & 399 & No & 2400 & 8200 & 0.60 & $2508 \pm 70$ \\
\hline
\end{tabular}

${ }^{a}$ Narrowest linewidths achieved with 852 and $780 \mathrm{~nm}$ lasers and their corresponding diode and grating parameters as defined in Table 1.

This result also agrees with the findings of Binder et al., i.e., lasers with different front facet reflectivities exhibit similar linewidths as long as they are in single-mode operation. ${ }^{17}$

The linewidth error of $80 \mathrm{kHz}$ is estimated from the fact that the amount of current injected into the diode laser influences the extent to which the laser operates in a single mode, which in turn affects the linewidth (Fig. 5). When the laser is about to jump to a different mode, it becomes slightly multi-mode, and the linewidth increases by an order of magnitude due to mode competition. Although we have verified that the laser operated in a single mode during our frequency noise measurements, Fig. 5 shows that there is still a current-dependent linewidth variation of $\sim 80 \mathrm{kHz}$ or $20 \%$ in the single-mode regime.

Table 3 summarizes the smallest linewidths measured for the near-IR diode lasers $(780,852 \mathrm{~nm})$. For comparison, the linewidth of the near-UV laser $(399 \mathrm{~nm})$ is included.

\section{Mechanical Stability of Laser Mounts}

Figure 6 shows a new laser mount (mount B) that is designed to reduce mechanical oscillations at higher acoustic frequencies. For the regular mount (mount A, see Fig. 2), ${ }^{2}$ the grating and laser diode are separately attached to a third aluminum piece that serves as the base of the mount. The vertical and horizontal grating angles are adjusted by turning the screws of the mirror mount holding the grating. Conversely, for mount $\mathrm{B}$, the aluminum block containing the grating is attached directly to the block containing the laser diode via four nylon pull screws. Three stainless steel screws, which push on the block containing the grat-

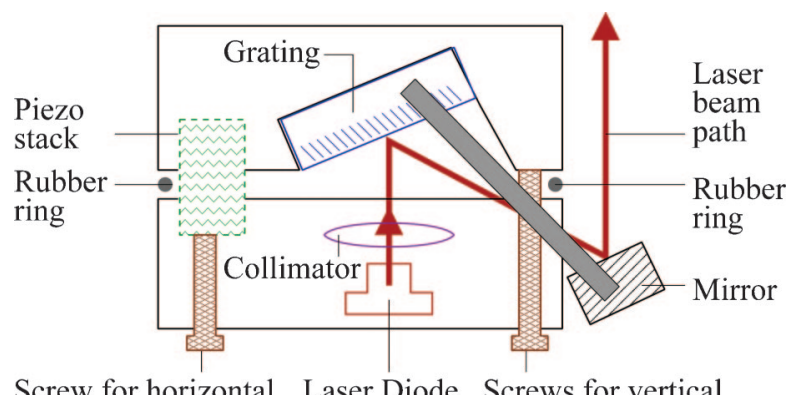

Screw for horizontal Laser Diode Screws for vertical grating alignment grating alignment

Fig. 6. (Color online) Schematic of mount B. The four nylon pull screws that attach the two aluminum blocks to each other are not drawn.

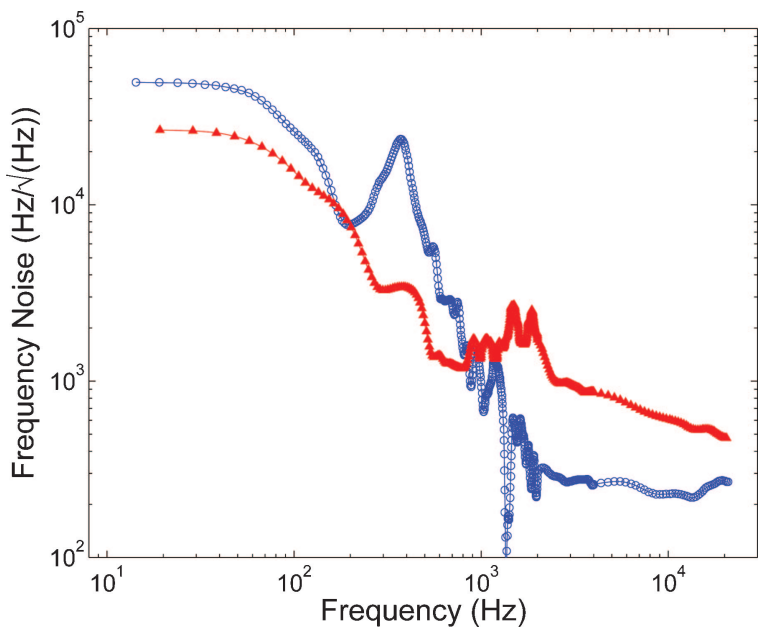

Fig. 7. (Color online) Frequency noise power spectral densities for mount B (open circles) and mount A (filled triangles) lasers. Mount $\mathrm{B}$ only has a strong mechanical resonance at $500 \mathrm{~Hz}$, whereas mount A has mechanical resonances at $2 \mathrm{kHz}$.

ing and a piezostack sandwiched between the two blocks, respectively, allow the vertical and horizontal grating angles to be adjusted. In fact, the vertical angle only needs to be adjusted slightly, because the optical feedback is already near optimal when the grating sits flush in the machined pocket. Mount B also includes a mirror, which couples the beam out of the mount in a fixed direction regardless of the grating's horizontal angle. ${ }^{30}$

The mechanical properties of mount B are characterized in terms of its frequency noise power spectral density and rms jitter at low Fourier frequencies (up to $5 \mathrm{kHz}$ ) and are plotted in Figs. 7 and 8, respectively. For comparison, Figs. 7 and 8 also display the data for a laser assembled with mount A. At first glance, mount A appears to be more stable than

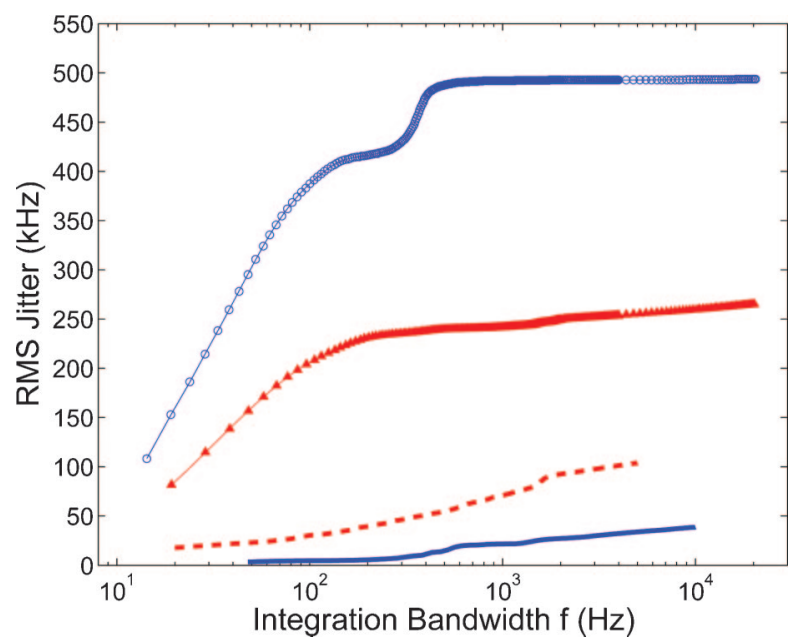

Fig. 8. (Color online) Rms jitters of lasers assembled both with mount B (open circles for unlocked and solid curve for locked) and mount A (filled triangles for unlocked and dashed curve for locked) versus integration bandwidth $f$. The $\mathrm{rms}$ jitter is given by $\Delta v_{\mathrm{jitter}}(f)=\left[\int_{0}^{f}\left(S\left(f^{\prime}\right)\right)^{2} \mathrm{~d} f^{\prime}\right]^{1 / 2}$. 
mount $\mathrm{B}$, because its overall noise is lower. However, the low-frequency noise can be easily reduced with a feedback circuit. In fact, the lower the frequencies at which mechanical resonances occur, the easier it is for the feedback circuit to compensate for noise. In this respect, mount $B$ is better than mount $A$ because its strong mechanical resonance only occurs at 500 $\mathrm{Hz}$, whereas mount $\mathrm{A}$ has strong resonances at $\sim 2$ $\mathrm{kHz}$. As shown in Fig. 8, the rms jitter of the laser built with mount $B$ is much lower than that for mount A, when both lasers are locked. Integrating from $0 \mathrm{~Hz}$ up to a bandwidth of $f=10 \mathrm{kHz}$, we achieve rms jitters of $\Delta v_{\text {jitter }}(f)=\left\{\int_{0}^{f}\left[S\left(f^{\prime}\right)\right]^{2} \mathrm{~d} f^{\prime}\right\}^{1 / 2}=40$ and 100 $\mathrm{kHz}$ for the actively stabilized lasers built with mounts B and A, respectively.

\section{Conclusion}

We have measured frequency noise power spectral densities to determine laser linewidths for various grating laser setups. We find typical linewidths of $250-600 \mathrm{kHz}$ for lasers operating near 399,780 , and $852 \mathrm{~nm}$. The linewidth depends on the type of grating and collimator. The presence of an AR coating has an insignificant effect on the linewidth reduction but enhances tuning stability. On the other hand, the higher the grating reflectivity and grating resolution, the narrower the linewidth. In particular, the grating resolution has a larger effect on the linewidth than the grating reflectivity, indicating that a complete theory of external-cavity diode lasers has to account for line broadening due to mode competition.

Using a new laser mount with improved mechanical properties, we were able to achieve a rms jitter of $40 \mathrm{kHz}$ for an actively stabilized system with a loop bandwidth of only $1.5 \mathrm{kHz}$.

We gratefully acknowledge support from the National Science Foundation, Defense Advanced Research Projects Agency, and the U.S. Army Research Office. H. Loh thanks the John Reed Undergraduate Research Opportunities Program Fund, Josephine de Kármán Fellowship, and Agency for Science, Technology and Research (Singapore) Scholarship. J. Simon is supported by the U.S. Department of Defense through the National Defense Science and Engineering Graduate Fellowship.

\section{References}

1. C. E. Wieman and L. Hollberg, "Using diode lasers for atomic physics,” Rev. Sci. Instrum. 62, 1-20 (1991).

2. L. Ricci, M. Weidemüller, T. Esslinger, A. Hemmerich, C. Zimmermann, V. Vuletic, W. König, and T. W. Hänsch, "A compact grating-stabilized diode laser system for atomic physics," Opt. Commun. 117, 541-549 (1995).

3. H. Patrick and C. E. Wieman, "Frequency stabilization of a diode laser using simultaneous optical feedback from a diffraction grating and a narrowband Fabry-Perot cavity," Rev. Sci. Instrum. 62, 2593-2595 (1991).

4. R. Lang and K. Kobayashi, "External optical feedback effects on semiconductor injection laser properties," IEEE J. Quantum Electron. 16, 347-355 (1980).

5. S. Saito, O. Nilsson, and Y. Yamamoto, "Oscillation center frequency tuning, quantum FM noise, and direct frequency modulation characteristics in external grating loaded semiconductor lasers," IEEE J. Quantum Electron. 18, 961-970 (1982).

6. E. Patzak, H. Olesen, A. Sugimura, S. Saito, and T. Mukai, "Spectral linewidth reduction in semiconductor lasers by an external cavity with weak optical feedback," Electron. Lett. 19, 938-940 (1983)

7. E. Patzak, A. Sugimura, S. Saito, T. Mukai, and H. Olesen, "Semiconductor laser linewidth in optical feedback configurations," Electron. Lett. 19, 1026-1027 (1983).

8. G. P. Agrawal, "Line narrowing in a single-mode injection laser due to external optical feedback," IEEE J. Quantum Electron. 20, 468-471 (1984).

9. C. H. Henry, "Theory of spontaneous emission noise in open resonators and its application to lasers and optical amplifiers," J. Lightwave Technol. 4, 288-297 (1986).

10. H. Sun, S. Menhart, and A. Adams, "Calculation of spectral linewidth reduction of external-cavity strong-feedback semiconductor lasers," Appl. Opt. 33, 4771-4775 (1994).

11. K. Kikuchi and T. Okoshi, "Simple formula giving spectrumnarrowing ratio of semiconductor-laser output obtained by optical feedback," Electron. Lett. 18, 10-11 (1982).

12. R. F. Kazarinov and C. H. Henry, "The relation of line narrowing and chirp reduction resulting from the coupling of a semiconductor laser to a passive resonator," IEEE J. Quantum Electron. 23, 1401-1409 (1987).

13. B. Tromborg, H. Olesen, X. Pan, and S. Saito, "Transmission line description of optical feedback and injection locking for Fabry-Perot and DFB lasers," IEEE J. Quantum Electron. 23, 1875-1889 (1987).

14. M. W. Fleming and A. Mooradian, "Spectral characteristics of external-cavity controlled semiconductor lasers," IEEE J. Quantum Electron. 17, 44-59 (1981).

15. R. Wyatt and W. J. Devlin, " $10 \mathrm{kHz}$ linewidth $1.5 \mu \mathrm{m}$ InGaAsP external cavity laser with $55 \mathrm{~nm}$ tuning range," Electron. Lett. 19, 110-112 (1983).

16. R. Wyatt, "Spectral linewidth of external cavity semiconductor lasers with strong, frequency-selective feedback," Electron. Lett. 21, 658-659 (1985).

17. J. O. Binder, G. D. Cormack, and A. Somani, "Intermodal tuning characteristics of an InGaAsP laser with optical feedback from an external-grating reflector," IEEE J. Quantum Electron. 26, 1191-1199 (1990).

18. G. Genty, A. Gröhn, H. Talvitie, M. Kaivola, and H. Ludvigsen, "Analysis of the linewidth of a grating-feedback GaAlAs laser," IEEE J. Quantum Electron. 36, 1193-1198 (2000).

19. G. Genty, M. Kaivola, and H. Ludvigsen, "Measurements of linewidth variations within external-cavity modes of a gratingcavity laser," Opt. Commun. 203, 295-300 (2002).

20. I. D. Henning, "Linewidth broadening in semiconductor lasers due to mode competition noise," Electron. Lett. 19, 935-936 (1983).

21. H. Talvitie, A. Pietiläinen, H. Ludvigsen, and E. Ikonen, "Passive frequency and intensity stabilization of extendedcavity diode lasers," Rev. Sci. Instrum. 68, 1-7 (1997).

22. C. Audoin, "Frequency metrology," in Metrology and Fundamental Constants, corso 68 of International School of Physics Enrico Fermi, A. F. Milone, P. Giacomo, and F. Leschiutta, eds. (North-Holland, 1980), pp. 169-222.

23. $\mathrm{H}$. Li and H. R. Telle, "Efficient frequency noise reduction of GaAlAs semiconductor lasers by optical feedback from an external high-finesse resonator," IEEE J. Quantum Electron. 25, 257-264 (1989).

24. T. Laurila, T. Joutsenoja, R. Hernberg, and M. Kuittinen, "Tunable external-cavity diode laser at $650 \mathrm{~nm}$ based on a transmission diffraction grating," Appl. Opt. 41, 5632-5637 (2002).

25. T. Kiguchi, A. Uematsu, M. Kitano, and H. Ogura, "Grating external cavity diode lasers with broad tunable range and 
narrow spectral linewidth for high-resolution spectroscopy," Jpn. J. Appl. Phys., Part 1 35, 5890-5895 (1996).

26. K. L. Corwin, Z.-T. Lu, C. F. Hand, R. J. Epstein, and C. E. Wieman, "Frequency-stabilized diode laser with the Zeeman shift in an atomic vapor," Appl. Opt. 37, 3295-3298 (1998).

27. B. Dahmani, L. Hollberg, and R. Drullinger, "Frequency stabilization of semiconductor lasers by resonant optical feedback," Opt. Lett. 12, 876-878 (1987).
28. C. H. Henry, "Theory of the linewidth of semiconductor lasers," IEEE J. Quantum Electron. 18, 259-264 (1982).

29. M. W. Fleming and A. Mooradian, "Fundamental line broadening of single-mode ( $\mathrm{GaAl})$ As diode lasers," Appl. Phys. Lett. 38, 511-513 (1981).

30. C. J. Hawthorn, K. P. Weber, and R. E. Scholten, "Littrow' configuration tunable external cavity diode laser with fixed direction output beam," Rev. Sci. Instrum. 72, 4477-4479 (2001). 